DOI: $10.1007 / \mathrm{s} 00350-015-4178-9$

\section{Kostensensible Leitlinien.}

Herausgegeben von Georg Marckmann. Medizinisch Wissenschaftliche Verlagsgesellschaft, Berlin 2015, 209 S., kart., $€ 39,95$

In dem von Marckmann herausgegebenen, auf Ergebnissen des vom Bundesministerium für Bildung und Forschung geförderten interdisziplinären Forschungsverbunds „Allokation“ basierenden Sammelband gehen zwölf Autoren aus verschiedenen Fachrichtungen - darunter Mediziner, Medizinethiker, Gesundheitsökonomen und Medizinrechtler - in zehn Beiträgen aus unterschiedlichen Perspektiven der Frage nach, welche Rolle sog. Kostensensible Leitlinien (KSLL) als Instrumente einer expliziten, evidenzbasierten Leistungssteuerung für eine effiziente und gerechte Gesundheitsversorgung spielen können. Ausgangspunkt ist dabei die weithin geteilte Annahme, durch medizinische Innovationen und den demographischen Wandel werde sich die Finanzsituation im deutschen Gesundheitswesen in den kommenden Jahren weiter verschärfen. Leistungseinschränkungen in der Gesundheitsversorgung sind damit auf Dauer unvermeidbar. Offen ist jedoch, wie genau in einer medizinisch rationalen, ökonomisch sinnvollen und ethisch wie rechtlich vertretbaren Weise mit den begrenzt verfügbaren Ressourcen im Gesundheitswesen umgegangen werden kann. Diesbezüglich will die Forschergruppe Perspektiven aufzeigen. Evidenzbasierten Allokationsentscheidungen werde eine immer größere Bedeutung zukommen, wobei diese sich bereits aus gerechtigkeitsethischen Gründen an klar definierten Verfahren und Kriterien zu orientieren haben. Implizite Leistungsbegrenzungen, bei denen der Arzt ohne weitere Vorgaben im Einzelfall über die Verteilung begrenzter Gesundheitsleistungen entscheidet, begegnen insofern naturgemäß gewichtigen Bedenken. Bislang fehlen jedoch konkrete Vorschläge, wie eine explizite Leistungssteuerung in der Praxis erfolgen kann.

An dieser Stelle setzt das vorgestellte Konzept der KSLL an. Medizinische Leitlinien sind in der Praxis als Orientierungspunkte ärztlicher Tätigkeit generell von erheblicher Bedeutung. Nach allgemeinem Verständnis handelt es sich hierbei um systematisch entwickelte, wissenschaftlich begründete und praxisorientierte Entscheidungshilfen für die angemessene ärztliche Vorgehensweise bei speziellen gesundheitlichen Problemen. Sie bedürfen freilich stets der kritischen Uberprüfung in der konkreten Behandlungssituation und sind rechtlich nicht ohne Weiteres verbindlich. Fraglich ist nun, ob und wie Kostenerwägungen sinnvoll in diese grundsätzlich rein medizinisch geprägten Handlungsanweisungen integriert werden können, um dem Arzt auch in dieser Hinsicht Entscheidungshilfen für den Einzelfall an die Hand zu geben. Erklärtes Ziel der KSLL ist dabei keine generelle Absenkung des Leistungsniveaus zur Kostenersparnis, vielmehr sollen unausweichliche Leistungsbegrenzungen so differenziert durchgeführt werden, dass den Patienten lediglich ein möglichst geringer (Zusatz-)Nutzen vorenthalten wird.

Wiss. Mitarb. Christoph Jansen, LL.M., Institut für Medizinrecht, Universität zu Köln, Köln, Deutschland
Eingangs schildern Strech/Marckmann aktuelle empirische Befunde zum Umgang mit begrenzten Mitteln in deutschen Krankenhäusern. Bereits heute sind demnach Allokationsentscheidungen im Klinikalltag allgegenwärtig. Im Anschluss geht Marckmann ausführlich auf die ethischen Grundlagen der Debatte um KSLL ein. Sodann wird aus gesundheitsökonomischer Sicht in die Methodik der Erstellung von KSLL eingeführt. Das Werk beschränkt sich dabei jedoch nicht auf abstrakte Überlegungen, vielmehr werden in zwei ausgewählten Praxisbereichen (Kardiologie und Intensivmedizin) die Möglichkeiten und Grenzen einer expliziten Leistungssteuerung anhand konkreter Beispiele untersucht und hierfür jeweils exemplarische KSLL entwickelt. Die Erläuterungen zur Leitlinienerstellung sowie die Kurzfassungen der erarbeiteten KSLL (samt Einführung in das Grundkonzept der KSLL und für den medizinischen Laien leichter verständlicher Versionen) tragen in besonderem Maße zur Veranschaulichung der Problematik bei. Zudem wurden sowohl Entscheidungsträger und Interessenvertreter aus dem Gesundheitswesen als auch Patienten nach ihren Einschätzungen zu Stärken und Schwächen der KSLL gefragt, die Ergebnisse dieser Studien werden in den beiden abschließenden Beiträgen präsentiert.

Aus juristischer Sicht von besonderem Interesse sind die Beiträge von Huster/Held und Hauck, in denen eingehend die sozialrechtlichen Rahmenbedingungen einer expliziten Leistungssteuerung durch KSLL als Rationierungsinstrumente in der gesetzlichen Krankenversicherung untersucht und de lege lata bestehende Bedenken aufgezeigt werden. Hingewiesen sei in diesem Zusammenhang darauf, dass die Ressourcenknappheit im Gesundheitswesen auch das Zivilrecht vor erhebliche Herausforderungen stellt. Der steigende Kostendruck lässt ein Spannungsverhältnis von Haftungs- und Sozialrech befürchten, sobald die haftungsrechtlichen Mindestanforderungen an die Behandlung die Maximalstandards der sozialrechtlichen Kostentragung übersteigen. Es stellt sich daher in Zukunft ebenfalls die Frage, welchen Einfluss KSLL auf den medizinischen Standard als Maßstab der ärztlichen Behandlungsfehlerhaftung nehmen können (zur Bedeutung von Leitlinien für den haftungsrechtlichen Standardbegriff s. Katzenmeier, in: Laufs/Katzenmeier/Lipp, Arztrecht, 7. Aufl. 2015, Kap. X, Rdnrn. 10 f.; zu Kostendruck und Standard Rdnrn. $21 \mathrm{ff}$.).

Das Werk richtet sich - dem interdisziplinären Ansatz entsprechend - ausdrücklich an eine breite Zielgruppe: an Wissenschaftler und Praktiker in der Gesundheitsversorgung, in Krankenkassen, in der Gesundheits- und Wirtschaftspolitik, im öffentlichen Gesundheitswesen, in Dienstleistungs- und Industrieunternehmen der Gesundheitsbranche, an Gesundheitsökonomen, Medizinmanager, Betriebs- und Volkswirte, Gesundheitswissenschaftler, an Ärzte in der Wissenschaft, Klinik und Praxis, an Studenten der Medizin und Gesundheitswissenschaften, aber auch an Patienten, Betroffene und Angehörige. Es ist darüber hinaus für Juristen aus Wissenschaft und Praxis mit medizin- und gesundheitsrechtlichem Tätigkeitsschwerpunkt uneingeschränkt empfehlenswert. Insgesamt liefert der Sammelband viele wertvolle Beiträge zu einer zentralen Zukunftsfrage des deutschen Gesundheitssystems und präsentiert hierfür in Form der Leistungssteuerung durch KSLL ein konkretes Lösungskonzept, welches dem eigenen Anspruch des Werks, eine kritische Auseinandersetzung mit diesem Ansatz zu ermöglichen und so zur Diskussion über den vernünftigen Einsatz begrenzt verfügbarer Ressourcen im Gesundheitswesen beizutragen, ohne Weiteres gerecht wird. 